\title{
Controversies around Xylitol
}

Xylitol research has been conducted since the early 70's; at the beginning of 2009 there were almost 500 PubMed- indexed papers on "xylitol and dental". Xylitol research was initiated in Finland, but nowadays the majority of papers are published by non-Finnish authors. Xylitol studies have been criticized mainly by European researchers. This criticism is reflected for example in the most recent textbook of cariology, Dental Caries - The Disease and its Clinical Management (2008), used by students in all Nordic countries. It shows a figure with reduction in caries occurrence in the Turku Sugar Studies as an example, not of the effect of total substitution of dietary sugars with xylitol, but rather "indicating the importance of removal of sugar from the diet, rather than starch, in caries control“. Moreover, a frequently cited review in Caries Research in 2004 came to the conclusion that "there is no evidence for a cariestherapeutic effect of xylitol". 'Xylitol studies are easy to criticize especially by authors who have never been involved in field trials. For example, to avoid exchange of test products between children of the same class randomisation has to be done by schools or school classes. This is rarely recognized by the critics of xylitol studies. In addition, it is hard to find volunteers to comply with 2-3 years chewing of a hard and tasteless gum base, which is the control often suggested for chewing gum trials. Polyols like maltitol and sorbitol are by no means inert and are thus not valid controls for xylitol. Fortunately, US dental experts have critically, but positively, conducted research and reviewed the literature on xylitol. ${ }^{2-5}$

One consequence of practical problems associated with field trials is that in systematic reviews of methods for caries control and prevention the strict exclusion criteria leave only few xylitol studies for evaluation. For example, the inclusion criteria for a Swedish systematic review on methods of caries prevention (SBU; www.sbu.sel were so strict that hardly any recommendations concerning caries prevention could be made. This also applied to xylitol: according to the report, the evidence on the clinical efficacy for xylitol was inconclusive. In a critical evaluation of the SBU report, the following question was asked "where does this leave the confused dental practitioner, who has to offer chairside advice to patients, but cannot wait of further (good-quality) research to be conducted and published, providing a more solid evidence-base?" (Stillman-Lowe, 2005). On the other hand, xylitol has been recommended for use in caries control and prevention by several dental associations. Also the European Food Safety Authority evaluated the literature on xylitol in 2008 and came to the conclusion that "xylitol chewing gum reduces the risk of caries in children". The claim had to be reformulated since chewing gum is not a medicine and can thus not be claimed to reduce the risk of a disease, caries.

In Europe, there is a strong interest not to give xylitol a better status than for other polyol sweeteners. Xylitol differs from other polyols as is obvious to all experts familiar with microbiology. Xylitol has beneficial effects on the oral flora not shared by other polyols. The evidence so far supports specific xylitol-effects on oral bacteria, but not on saliva. Xylitol cannot be metabolized by plaque bacteria, contrary to sorbitol and other 6-carbon polyols, and may thus favour mineralization. The accumulated evidence suggests that 1) mutans streptococci are the target organisms of xylitol in vivo, 2) mutans streptococci are reduced and stay on a lower level even during long-term xylitol consumption as long as the consumption lasts, 3) xylitol consumption reduces the accumulation of plaque, 4) xylitol consumed by mothers reduces the mother-child transmission of mutans streptococci and consequently, caries occurrence in their children, 6,7 and 5) xylitol consumption reduces the risk of caries in children. ${ }^{4}$ In clinical trials on xylitol mostly 
chewing gum with high xylitol concentrations has been used.

Studies with low daily doses of xylitol are known as a rule to fail to show any cariespreventive effects. To obtain beneficial xylitoleffects on mutans streptococci, on plaque and caries occurrence xylitol must be consumed three times or more per day, with a daily dose of 5-6 grams. 4,7 When using chewing gums or pastilles, saliva stimulation always occurs due to chewing or sucking. Some authors have claimed that the beneficial effects of xylitol are solely based on saliva stimulation and that all sugarfree gums are similar in this respect. In fact, in conditions where caries occurrence is high, even sorbitol gum can prevent caries. ${ }^{8}$ A recent study elegantly shows caries prevention with xylitol without any saliva stimulation. In this study fifteen-month-old children were given xylitol or sorbitol syrup with a syringe for 12 months. ${ }^{9}$ The xylitol syrup prevented early childhood caries effectively, while sorbitol had no effect.

In spite of the abundant literature still more studies are needed on the action of xylitol. Biofilm models could be very useful in xylitol research. There is a clear need for properly designed, randomized, controlled, clinical trials to demonstrate 1) the feasibility of xylitol prevention in different populations with different dietary and oral hygiene habits, 2) suitable vehicles to deliver xylitol, 3) the extent to which xylitol can be "diluted" with other polyols without losing the caries preventive effects, and 4) the minimum daily xylitol dose and frequency of xylitol are needed to obtain the expected xylitoleffects on mutans streptococci, plaque and most importantly, on caries occurrence.

Clinical trials often aim to improve treatment methods. When several clinical trials give similar results, clinicians can be given treatment guidelines based on findings verified by research. In this way research serves the clinicians in treating patients according to evidence-based solid information. Systematic reviews are currently required to update guidelines for caries prevention. As such, critical evaluation of the existing literature is a positive goal, but if it leads to a situation where no treatment guidelines can be given, something is wrong. This also applies to xylitol studies. Though strict inclusion criteria prevent conclusions based on systematic reviews of the literature, evidencebased treatment guidelines can still be given for the use of xylitol. Xylitol is a useful adjunct to traditional methods for caries control and prevention. Caries prevention with xylitol has been claimed to be expensive, but if true primary prevention is obtained, as demonstrated in the mother-child study, ${ }^{6,7}$ it may be worth it.

\section{REFERENCES}

1. van Loveren C. Sugar alcohols: what is the evidence for caries-preventive and caries-therapeutic effects? Caries Res 2004;38:286-293.

2. Burt BA. The use of sorbitol- and xylitol-sweetened chewing gum in caries control. JADA 2006;137:190-196.

3. Featherstone JD. Delivery challenges for fluoride chlorhexidine and xylitol. BMC Oral Health 2006;61Suppl 1):S8.

4. Ly KA, Milgrom $P$, Rothen M. Xylitol, sweeteners, and dental caries. Pediatr Dent 2006;28:154-163.

5. Zero D. Are sugar substitutes also anticariogenic? JADA 2008;139:9S-10S.

6. Söderling $E$, Isokangas $P$, Pienihäkkinen $K$, Tenovuo J. Influence of maternal xylitol consumption on acquisition of mutans streptococci by infants. J Dent Res 2000;79:882887.

7. Söderling E. Xylitol, mutans streptococci, and dental plaque. Adv Dent 2009; in press.

8. Szöke J, Banoczy J. Effect of after-meal sucrose-free gumchewing on clinical caries. SADJ 2005;60:248-251.

9. Milgrom P, Ly KA, Tut OK, Mancl L, Roberts MC, Briand K, Gancio MJ. Xylitol pediatric topical syrup to prevent dental caries: a double blind, randomized clinical trial of efficacy. Arch Pediatr Adolesc Med 2009; in press. 